\title{
The impact of geographic mobility on the spread of COVID-19 in Hong Kong
}

\author{
Paulina Pui Yun Wong, ${ }^{1,2}$ Chien-Tat Low, ${ }^{3}$ Poh-Chin Lai ${ }^{3}$

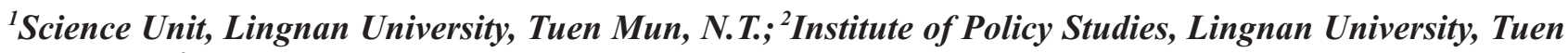 \\ Mun, N.T.; ${ }^{3}$ Department of Geography, Faculty of Social Sciences, The University of Hong Kong, Pokfulam, \\ Hong Kong SAR, China
}

\begin{abstract}
The modern highly globalised economy is jeopardising human health as the increased mobility and interconnectedness has the potential to rapidly transmit pathogens across the globe. This was recently confirmed by the coronavirus disease 2019 outbreak, which quickly led to localised outbreaks in virtually every country. As the existing health systems were unprepared, the world has witnessed a critical shortage of life-supporting and health-sustaining resources. In the absence of effective non-pharmaceutical interventions to suppress the virus transmission, many governments imposed total or partial lockdowns, with devastating economic consequences. The case of Hong Kong in quickly suppressing the virus from spreading can thus be a lesson for all. In this study, open data sources of infected individuals are employed to compile maps of disease incidents at various geographic scales with the aim of better understanding the transmission dynamics and discern spatial variability. Our findings show that tracking human mobility patterns can improve awareness of spatiotemporal factors driving the risks of human exposure to viruses. Moreover, we have demonstrated that spatial tools can be successfully employed to explore connections between individuals and wider communities with the aim of informing adaptation of policies at
\end{abstract}

Correspondence: Paulina Pui Yun Wong, Science Unit, Lingnan University and Institute of Policy Studies, Lingnan University, Rm116, Lau Chung Him Building, 8 Castle Peak Road, Tuen Mun, Hong Kong 10. Tel.: 852.93182944. E-mail: paulinawong@ln.edu.hk

Key words: COVID-19; disease mapping; mobility; spatial analysis; visualisation.

Acknowledgements: the authors would like to thank research team at the Science Unit of Lingnan University for crowdsourcing the necessary data to the study.

Received for publication: 22 May 2021.

Revision received: 29 November 2021.

Accepted for publication: 3 December 2021.

CCopyright: the Author(s), 2022

Licensee PAGEPress, Italy

Geospatial Health 2022; 17(s1):1022

doi:10.4081/gh.2022.1022

This article is distributed under the terms of the Creative Commons Attribution Noncommercial License (CC BY-NC 4.0) which permits any noncommercial use, distribution, and reproduction in any medium, provided the original author(s) and source are credited. different spatial scales and for different time periods. As was shown in the case of Hong Kong, disease control encompasses the interrelated tasks of reducing social interactions and encouraging adoption of protective behaviours.

\section{Introduction}

Increasing globalisation and transboundary movements are major causes of disease propagation (McMichael and Beaglehole, 2000), as evident from the rapid rate at which coronavirus disease 2019 (COVID-19) spread around the globe. While the rest of the world is still combating the seemingly unstoppable epidemic, Hong Kong has recorded relatively few cases compared with other densely populated cities due to quick action at the beginning of the outbreak, which has resulted in a consistent decline in local transmission rates as well as the mean effective reproductive number between 23 January 2020 and 12 May 2021 (He et al., 2020; School of Public Health, The University of Hong Kong, 2020); with little/no local infections thereafter. Many have speculated that the experience gained by the Hong Kong residents during the severe acute respiratory syndrome (SARS) epidemic in 2003 has prepared them well in terms of their adaptive routines and strict observation of hygiene measures, such as mask wearing and hand washing (Hung, 2003; Smith, 2006; McCloskey and Heymann, 2020). These behaviours are routinely practiced and accepted in Hong Kong and other Asian cities but are yet to be widely adopted in countries battered by COVID-19.

Although total lockdown was never imposed, despite being a common practice elsewhere (Alvarez et al., 2020; Lau et al., 2020), the number of travellers to Hong Kong by land and air transport decreased dramatically due to the travel bans imposed by other countries. Soon after the Chinese New Year break in early February 2020, the Government of Hong Kong also urged companies and businesses to allow their employees to work from home to curb the potential infection spread.

These intervention measures operated at three levels: individual, specific groups (such as pub/bar patrons), and Hong Kong as a whole. The protocols during the early stages of the epidemic required mandatory contact tracing of all infected/potentially infected individuals, immediate quarantine of infected individuals, and strict observance of mask wearing and hand hygiene by every resident. Border closure and suspension of public services and schools soon followed.

As is it now widely established that disease transmission is possible before the onset of any symptoms (He et al., 2020), contact tracing 2-14 days prior to case confirmation becomes crucial to identify the likely exposure locations. Geographic analysis through tracking of human mobility patterns can help focus attention on 
areas most at risk and prioritise actions to restrain disease spread. Given that the distribution of incidents across geography is not random, mapping the locations where incidents occur and comparing these locations with other factors such as time and other geographic features, areas of abnormal frequency can be highlighted to delineate problem areas and help optimise resources. Various geographic information system (GIS) and spatial statistical tools are available to quantify geographic variation patterns. For example, the hotspot analysis tool enables detecting locations of statistically significant disparities that account for the population sizes of both reference and target groups (Scott and Janikas, 2010). This kind of data reduction tool aids in pattern recognition by highlighting occurrence probability instead of co-location probability based on metric distance alone. In the context of disease mapping, these tools can be used to describe spatial variations in disease occurrence and characterise disease cases to identify potential etiological factors through visualisation (Lai et al., 2008; MacEachren, 2004).

The aim of this study is to examine the utility of spatial analysis and visualisation tools in examining disease incidents in a densely populated city during the early stages of outbreak, using Hong Kong as a case study. This is achieved by mapping mobility patterns of infected individuals using the GIS technology, open geospatial data of detailed contact tracing provided by the Government of the Hong Kong Special Administrative Region (HKSAR Government, 2020), and crowdsourcing data available on the news and social media. Potential areas of disease clusters can be detected through examining the distribution of destinations resulted from movement patterns. By comparing daily mobility indices (Citymapper, 2020) against the timeline of social distancing and other intervention measures, the effects of movement restriction policies implemented at different time intervals in suppressing disease outbreak can be studied. The study also presents different mapping techniques to visualise the association between disease distribution and transport infrastructures.

\section{Materials and methods}

\section{Study area and background}

Hong Kong is a special administrative region of China (Figure 1). Hong Kong is situated in the sub-tropical zone and has close socio-economic and cultural connections with Southern China and the Pearl River Delta. Hong Kong is one of the most densely populated megacities in the world, with about 7.4 million residents living within a small land area of only $1104 \mathrm{~km}^{2}$. Most of the city population resides in urbanised areas with densely packed highrise buildings and skyscrapers (comprising only $24 \%$ of its total land area), with is equivalent to 6890 individuals per $\mathrm{km}^{2}$. Hence, it is highly conducive to the spread of communicable diseases such as COVID-19.

\section{Data sources and compilation}

The Hong Kong Centre for Health Protection (CHP) is responsible for tracking disease development and treatment. CHP manages daily updates of confirmed and suspected cases and holds press conferences to address public concerns about surveillance criteria and related policies throughout the outbreak. Moreover, due to the open data policy in support of the development of Hong Kong into a smart city, anonymised official data, including demographic information and infection details are available online for public access. This study used disease incident data from 23 Jan to 6 May 2020 covering a total of 1041 confirmed cases. For each confirmed case, a date of onset is recorded and can be linked to all locations at which the person has resided, worked or visited in the past 14 days during the incubation periods, including their home/work addresses and with travel history covering airports, hotels, hospitals, clinics, and government-designated facilities. CHP also conducts contact tracing of infected individuals, especially for the locally transmitted cases to identify any potential clusters. Moreover, the anonymised data provided by CHP can be linked with detailed movement data crawled from Internet sources for the same period, including digital newspapers and social media networks. The procedure is necessary as contact tracing based on human recollection may have missed visits recorded on business sites and in between transits. By crawling the work locations and places of visits based on data from government sources, each confirmed case has on average three additional locations of movement over a period of fourteen days.

A distribution chart of mobility index was available from Citymapper (2020). It is a smartphone app which integrates volunteered geographic information for all urban modes of transport (including walking, cycling, and driving), in addition to public transportation (Vannoni et al., 2020). The mobility index reports volume of traffic and is expressed as 0 to 1 . If the index go above 1 , representing above average mobility, whereas below 1 , corresponding to lower than average mobility. As this app provides near real-time information about daily mobility patterns of Hong Kong, it can be used to compare the impacts of different policy interventions during the COVID-19 outbreak.

\section{Visualisation and mapping techniques}

Many interactive maps related to COVID-19 have been developed since the outbreak, whereby points are typically used to represent the location of individual cases (see HKSAR Government, 2020 ) or proportional circles to visualise the number of cases in certain areas (see Johns Hopkins, 2020). In these applications, incident is usually linked to the affected individual's home location. In the present study, this is replaced by the last residing address such as hospitals or designated quarantine hotels, in order to better connect with the local communities for emergency notification. The study used ArcGIS 10.8.1 (ESRI, 2020) for the compilation of spatial data and map visualisation.

The kernel density analysis tool (Carlos et al., 2010) can be employed to generate a density map which is a visually intuitive continuous surface showing areas with higher (lower) intensity of confirmed cases in darker (lighter) tones. The hotspot analysis tool takes the advantage of the neighbouring features (Scott and Janikas, 2010) to determine if an area is a statistically significant hot or cold spot. The tool also differentiates hot and cold spots into separate groups by varying confidence levels $(99 \%, 95 \%$, and $90 \%$ ). Whereas a hotspot represents an important location or a problem area requiring attention, it is worthwhile to examine a cold spot to explain its unique spatial characteristics or qualities different from the neighbouring features that might have contributed to the indifferent qualities. For example, a statistically significant cold spot may signify an area where protective services may not be needed, or such services may have already been implemented with satisfactory results. A cluster of cold spots can also help inform policymakers to relocate services to areas of higher need.

During the second outbreak in Hong Kong, a 'bar and music band' cluster was reported to be the largest and earliest cluster in 
the city involving 103 cases. Our analyses had more than 460 destination locations (including home, work, and places the individuals had shortly stayed) by extracting data released by the CHP and adding places the infected individuals had shortly visited or stayed through crowdsourcing from news reports and social media. A weighted kernel density map was generated by applying different weighting factors of 3:2:1 to home:work:stay respectively for barrelated infection clusters. We assumed that the risks of infection would be three times higher at home (with intimate contact and not wearing a mask), two times higher in the workplace (as co-workers would keep a social distance or wore a mask), compared to places of visit/stay (because of a shorter period of contact and with a mask on). The mapped results will vary with different weighting factors assigned with appropriate justification.

\section{Results}

In the present study, mapped results were examined to link geographic variations in disease dynamics with human mobility patterns and control measures during COVID-19 outbreak in Hong Kong.

\section{Assessing effectiveness of movement restrictions}

Table 1 provides a summary of confirmed COVID-19 cases in Hong Kong between 21 January and 06 May 2020. Confirmed cases are partitioned into six time blocks, showing higher local transmission rates in the early stages (A and B) which subsequently peaked (stages C-E). Majority of confirmed cases during the peak of the outbreak (periods D and E) were imported. The number of local transmissions peaked in period $\mathrm{D}$ and subsided in period $\mathrm{E}$ suggesting that intervention measures in the early stages was effective in slowing down local transmission in later stages.
Figure 2 shows a bar graph of the COVID-19 epidemic curve, partitioned into local and imported cases, plotted against the Citymapper mobility index represented as a black line. It also shows a timeline of movement restriction policies imposed in Hong Kong for the study period, with effective closure dates for public facilities (including indoor and outdoor recreational services, public libraries, and schools) and various businesses (such as pubs and bars, facial and massage parlours, and entertainment centres). As shown in Figure 2, the CHP imposed various containment and mitigation measures during the pandemic, most of which were short-term (usually valid for two weeks) with the possibility of further extensions. However, once these measures was relaxed slightly in early March, the second stage of the outbreak commenced. Heightened movement restriction measures were stringently enforced, leading to closure of select business outfits in early April until middle of May.

Table 1. A summary of confirmed cases stratified by imported and local transmissions.

\begin{tabular}{lcccc} 
Period & Period & $\begin{array}{c}\text { Total } \\
\text { Imported } \\
\text { cases }\end{array}$ & $\begin{array}{c}\text { Local } \\
\text { transmission }\end{array}$ \\
A & 23 Jan to 11 Feb & 49 & 13 & 36 \\
B & 12 Feb to 28 Feb & 45 & 8 & 27 \\
\hline C & 29 Feb to 16 Mar & 64 & 40 & 24 \\
D & 17 Mar to 02 Apr & 645 & 384 & 261 \\
\hline E & 03 Apr to 19 Apr & 223 & 159 & 64 \\
F & 20 Apr to 06 May & 15 & 14 & 1 \\
\hline
\end{tabular}

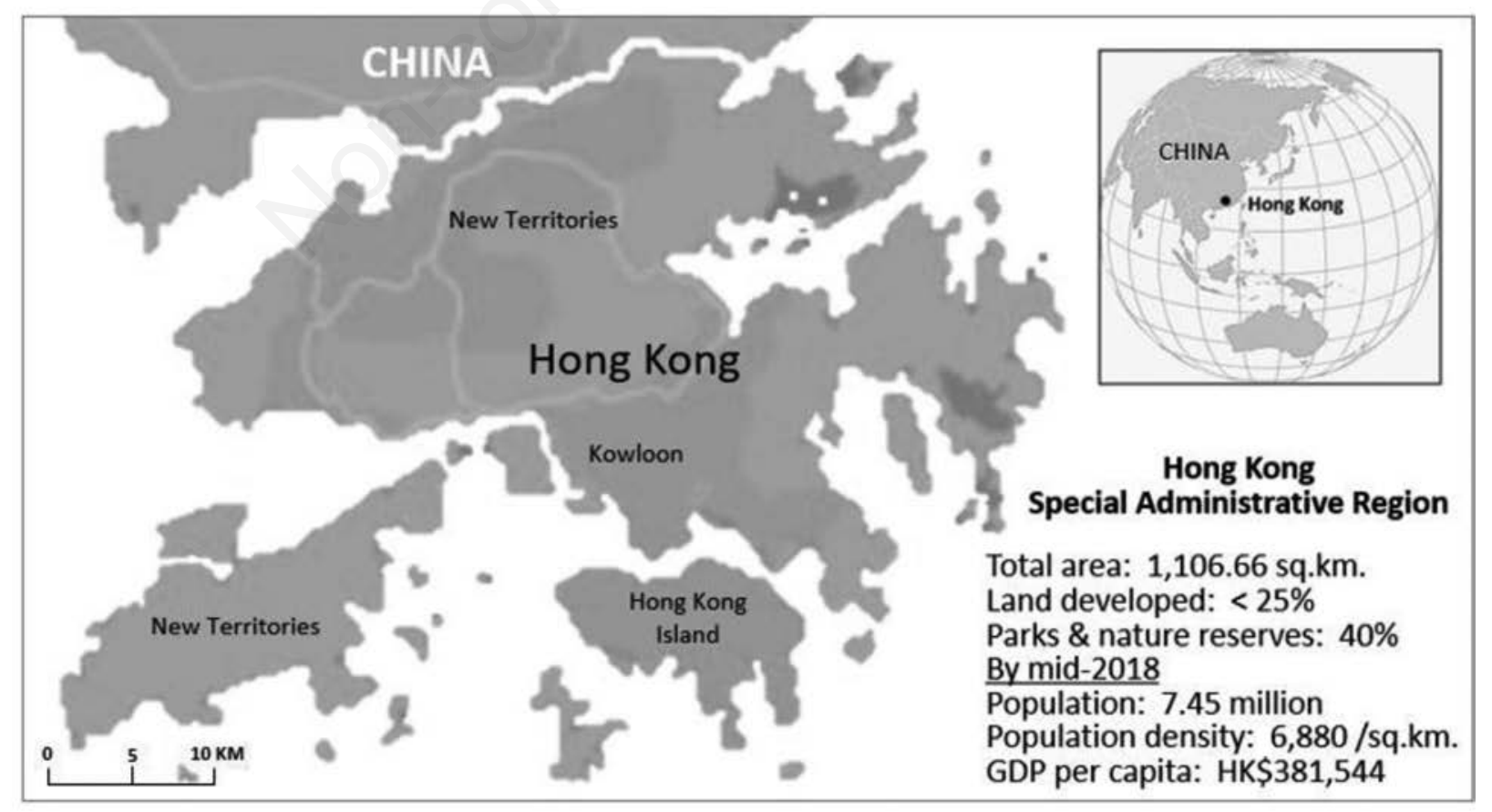

Figure 1. A location map of the Hong Kong Special Administrative Region. 
The inverse relationship between mobility and infection in Figure 2 suggests that human mobility and government-imposed social exclusion and isolation measures (indicated by arrows and timelines below the graph) seem to be closely linked. The graph shows that mobility declined rapidly as soon as the first confirmed case was reported on 23 January 2020. As the number of infections kept rising during period D (Figure 2), Hong Kong residents began to further restrict their mobility. However, once the number of new cases started to consistently decline, residents gradually resumed their everyday activities, albeit at a reduced rate compared to the levels before 23 January 2020. Prior to the report of the first confirmed case of COVID-19 (23 Jan 2020), the Citymapper mobility index of Hong Kong was assumed to be 1. Soon after the first reported case, school, non-essential work activities, and public services were suspended, resulting in a decline in the mobility index to below 0.6. During the second outbreak (denoted as period D in Figure 2), stricter policies and interventions were adopted due to a significant rise in the daily number of confirmed cases, and the index declined further to below 0.3. Hence, the movement restrictions (closure of workplaces, schools, and public services) have proven to be impactful, as the locally transmitted cases were soon under control, as evident from the rise in the mobility index to approx. $0.4-0.5$ of the original level.

\section{Detecting disease clusters and contact tracing}

The map of disease incidents shown in Figure 3 reveals that local and imported cases exhibited similar distributional patterns and concentrated in the most populated areas of Hong Kong. The disease concentration maps for the different epidemic periods shown below (denoted as A-F) further reveal that, in the earliest stage of the epidemic, a cluster of cases (circled in red) occurred in the northern part of Hong Kong where majority of immigrants from China have settled. This cluster was not visible in the remaining periods. It is also evident that the locations of highest disease concentration for each period remained relatively constant throughout the epidemic, with the exception of a wider spatial spread and higher intensity in period $\mathrm{D}$.

Figure 4A shows a weighted kernel density map generated by applying different weighting factors to home (purple), work (blue), and stay (green) for bar-related infection cluster. The results showed a widespread distribution of destinations with the sources of infection originating from five major bars across three regions (Tsim Sha Tsui, Central, and Wan Chai). Figure 4B displays results of the weighted density map. It is apparent that some hotspots (coloured in red and orange) in the New Territories were situated quite a distance from the bars or sources of infection. There were also several hotspots in Kowloon within localities of mixed commercial and residential developments. These spatially dispersed hotspots represented home locations of the pub/bar patrons. In addition, Wan Chai with two bars in which several infections were recorded is a true hotspot given that the district has mixed commercial and residential uses. Interestingly, two highly significant cold spots ( $99 \%$ confidence and coloured in blue) were found in Tsim Sha Tsui and Central, where three of the five bars identified as sources of infection were located. Both Tsim Sha Tsui and Central are business and commercial districts with very few residential establishments.

Figure 5 depicts the results of movement tracking and contact tracing of infected individuals along major transport routes. They were mostly band players, workers, and customers of the five bars identified as major sources of infection. It is evident from Figure 5

\section{Daily new imported and local cases in Hong Kong vs. Citymapper mobility index}

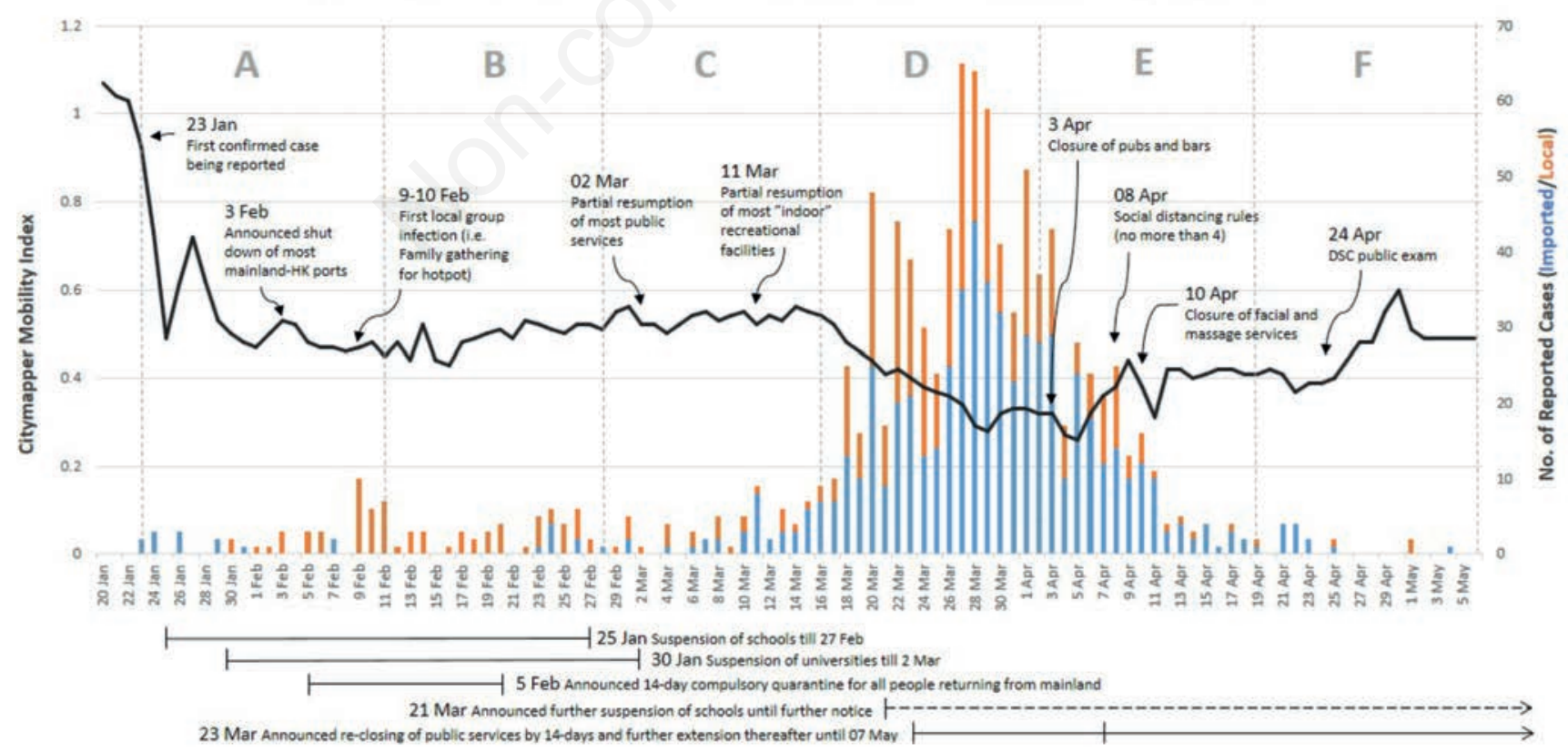

Figure 2. Daily new COVID-19 cases in Hong Kong (stratified by imported and local transmissions) versus Citymapper mobility index as a measure of the impact of the movement restrictions and interventions against the infection. 


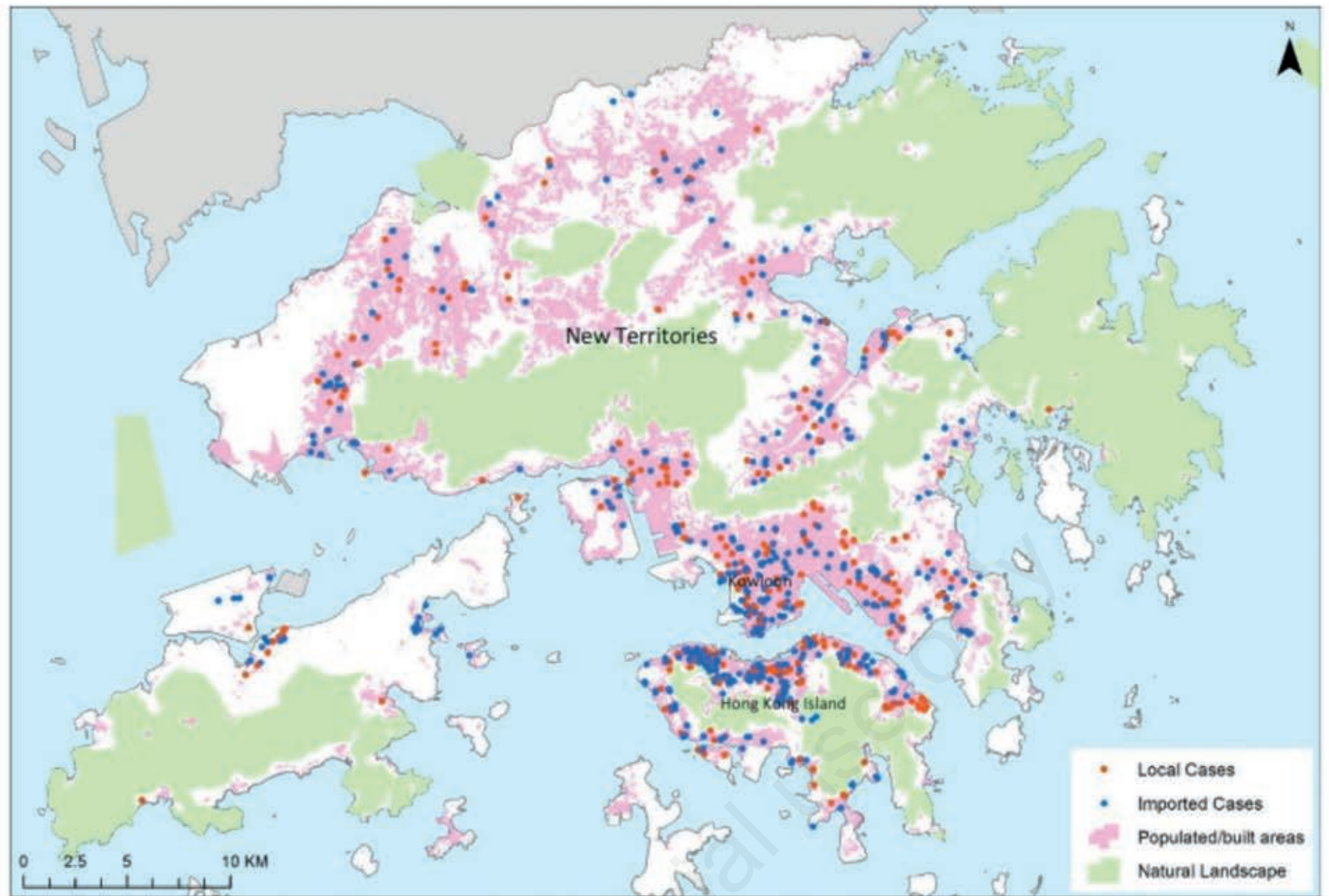

A

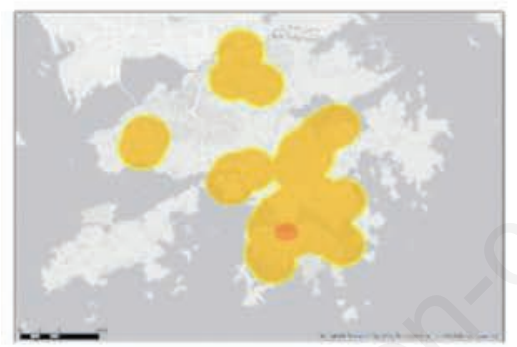

D

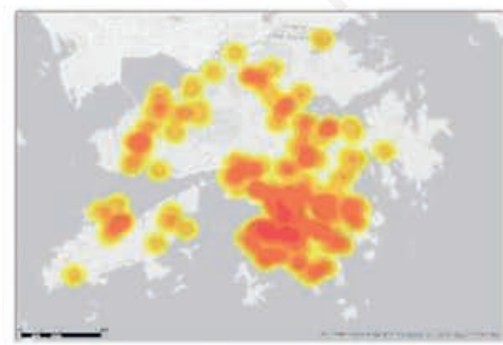

B

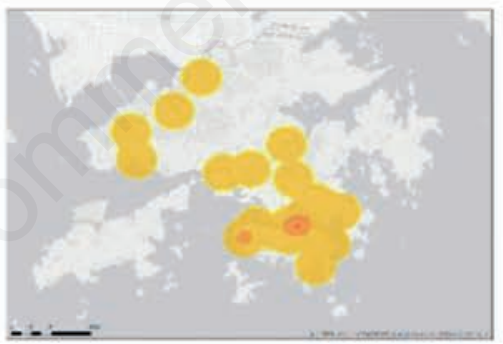

E

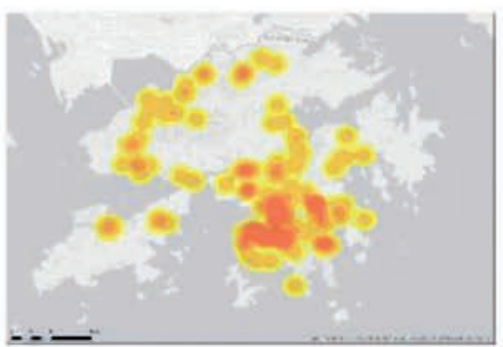

C

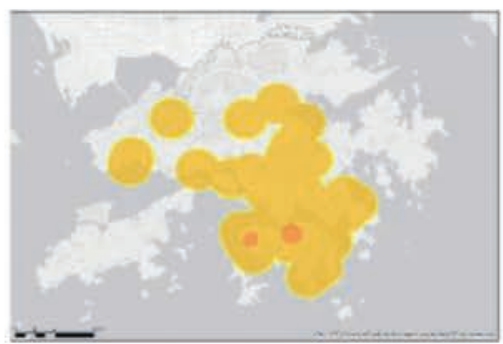

F

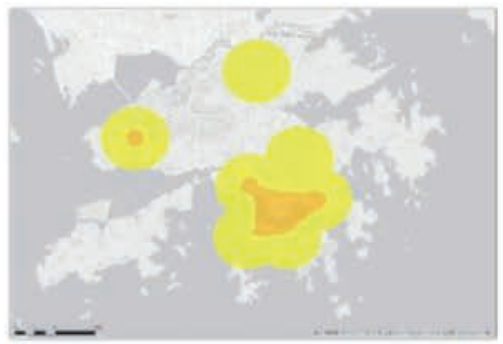

Kernel Density (17-day)

$\square<0.1$

$\square 0.1 \cdot 0.5$

$0.6 \cdot 1.0$

$\square 1.1 \cdot 5.0$

- $>5.0$

Figure 3. Spatial concentrations of confirmed cases by incident locations for six time periods shown in Figure 2. 
$\mathbf{A}$

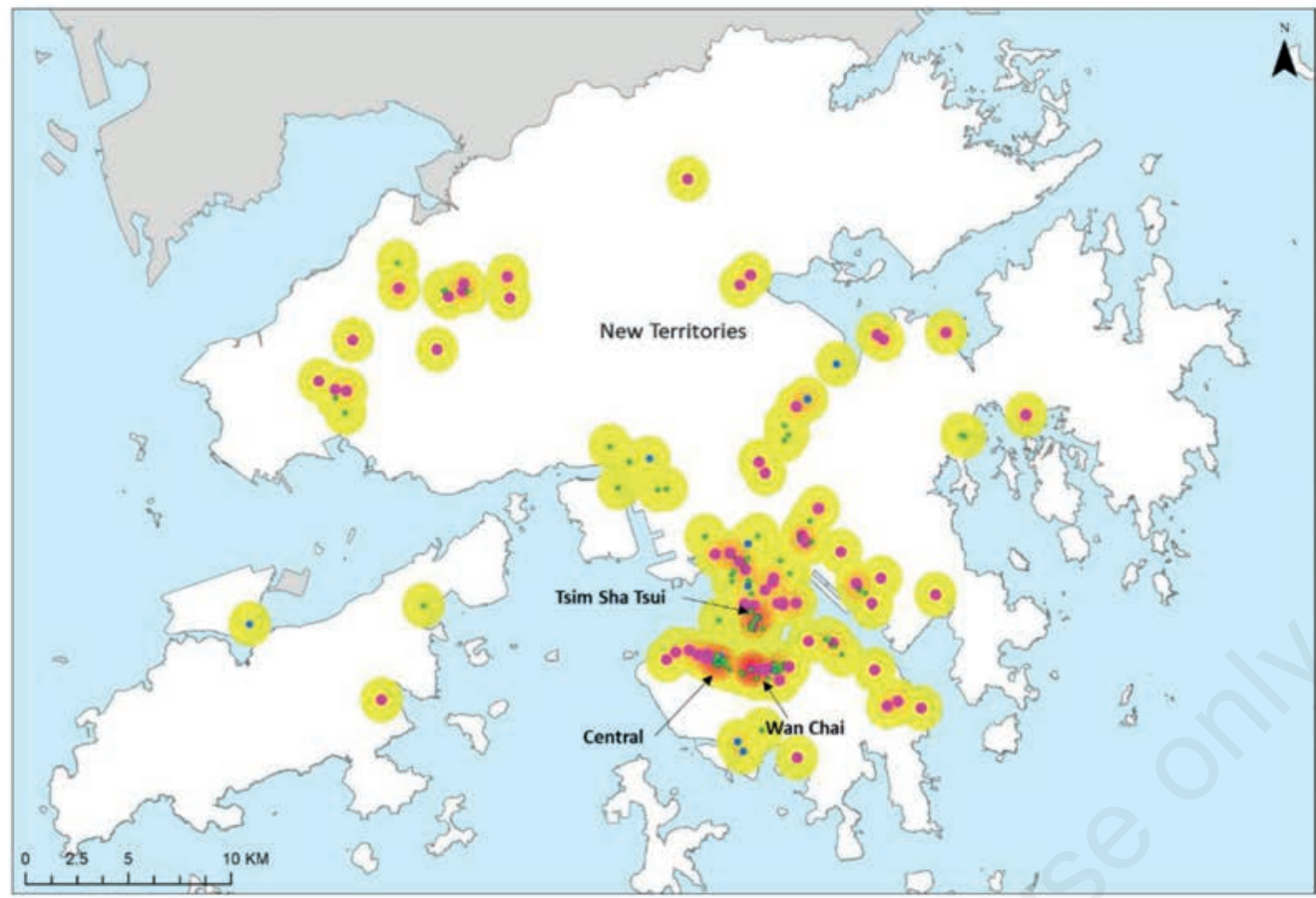

B

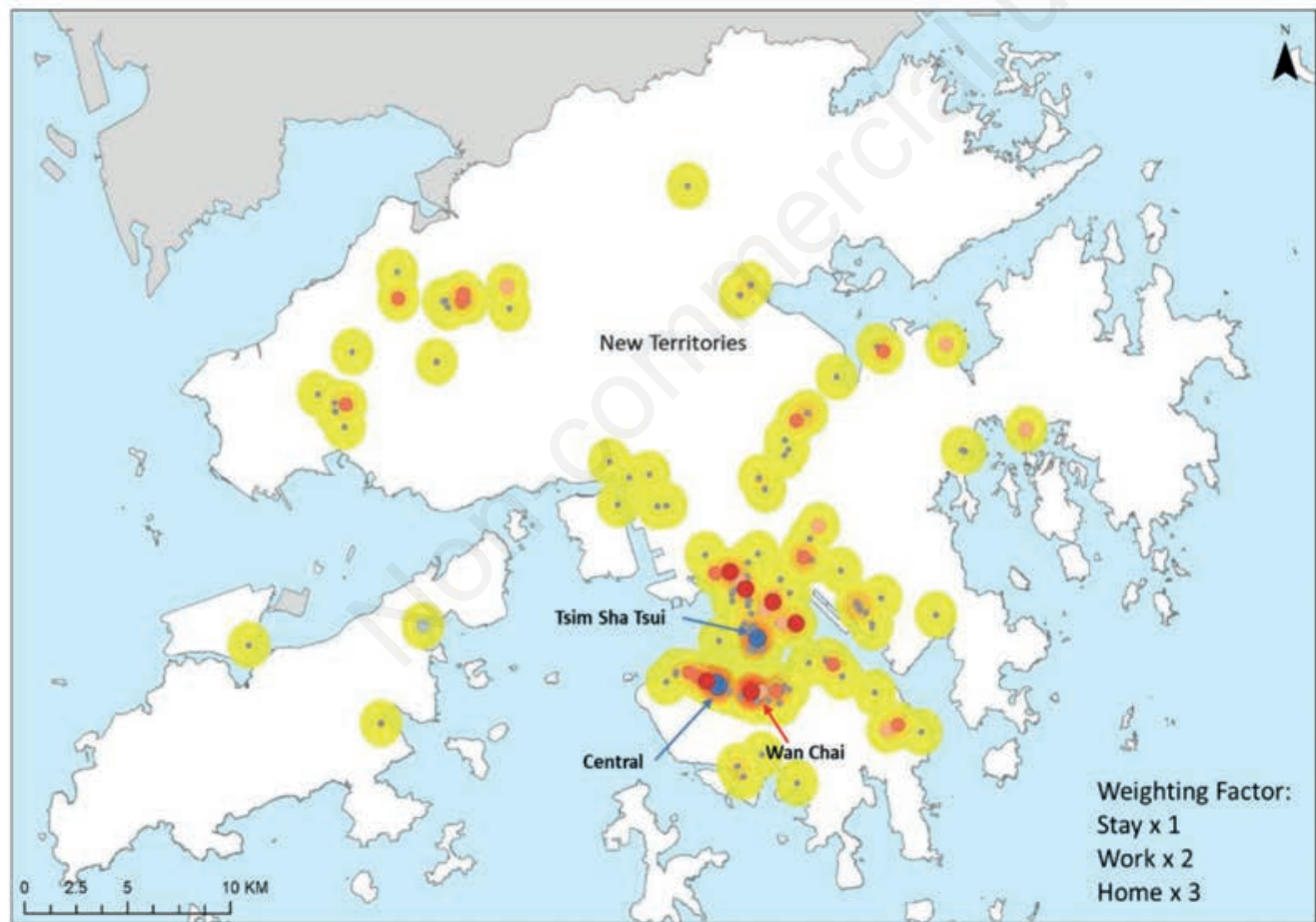

A Weighted Kernel Density Map with Bar-related Infection

Weighting Factor:

Stay $\times 1$

Work $\times 2$

Home $\times 3$

Travel Status

- Stay

- Work

- Home

Kernel Density (With Weight)

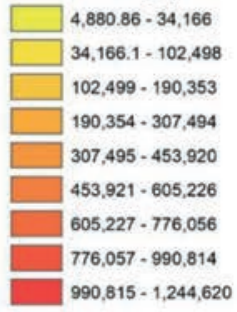

A hotspot map of bar-related infection

Larger symbol size for 99\% Confidence

Hotspot Analysis

Gi_Bin

- Cold Spot-99\% Confidence

Cold Spot - $95 \%$ Confidence

- Cold Spot - $90 \%$ Confidence

- Not Significant

- Hot Spot - $90 \%$ Confidence

- Hot Spot - $95 \%$ Confidence

- Hot Spot - $99 \%$ Confidence

Kernel Density (With Weight) $4,880.86-34,166$ $34,166.1$ - 102,498 $102,499-190,353$ $190,354-307,494$ 307,495 - 453,920 $453,921-605,226$ $605,227 \cdot 776,056$ $776,057 \cdot 990,814$ $990,815-1,244,620$

Figure 4. Hotspot analysis of bar-related COVID-19 infections in Hong Kong. A) A weighted kernel density map showing concentration of COVID-19 cases by spatial location. Home (purple), work (blue), and stay (green) locations are assigned different weighting factors. B) A hotspot map of bar-related infections revealing statistically significant hot and cold spots. 
that Wan Chai was responsible for disease outbreaks in neighbouring communities as well as for widespread transmission to other parts of Hong Kong. The widespread distribution could be a result not only of a large number of cases but also related to mobility behaviours of infected individuals.

\section{Discussion}

In the present study, we adopted a geographic perspective to examine disease pathways in order to develop a better understanding of the role of human mobility in shaping disease clusters. Disease transmission occurs through human contact and thus necessitates physical presence within a socio-spatial context. Due to the increasingly interconnected world through convenient transport linkages, the augmented potential for a large number of people to come into contact with infected individuals has become a reality. Hence, generating empirical insights into the interaction patterns under local conditions in relation to different control measures can help in the formulation of more effective public health policies.

\section{From SARS to COVID-19}

Many of the measures adopted in Hong Kong were also implemented in other countries, albeit with less success, as the CHP has benefitted from the experience gained during the SARS outbreak, allowing it to effectively differentiate imported from local infections (Figures 2 and 3). This action also entailed immediate and detailed contact tracing and prompt isolation of suspected cases, along with social distancing. In the absence of a vaccine and effective testing or treatment strategies, Hong Kong managed to keep the reproductive number low by quickly limiting human mobility and local interactions (HKU, 2020). These measures included working from home where possible, and closures of schools and other 'less-essential' public services.

As the 2003 SARS outbreak has shown, widespread acceptance of and strict compliance with imposed measures, including wearing facemasks in public places and improving personal hygiene, is essential in discouraging disease dissemination (Lai et al., 2004). As a result, Hong Kong residents quickly accepted this practice when the first COVID-19 cases emerged, given that wearing a surgical mask (as a protective gear against those who are sick or when one gets sick with a cold/flu) has become a norm. This was vital, given that transmission of this novel coronavirus could occur before an infected person develops symptoms, limiting the effectiveness of contact tracing.

Already on 31 December 2019, and as a component of emergency response in Hong Kong hospitals, all staff, patients, and visitors were required to wear masks and observe hand hygiene and environmental cleanliness (Wong et al., 2020). The heightened alertness has effectively controlled healthcare-associated and hospital-acquired COVID-19 infections. Figure 2 also shows the inverse relationships between mobility and infection and highlights the need to implement quarantine measures for inbound travellers, as seen in time period D. The graphs are simple to understand and useful for public information.

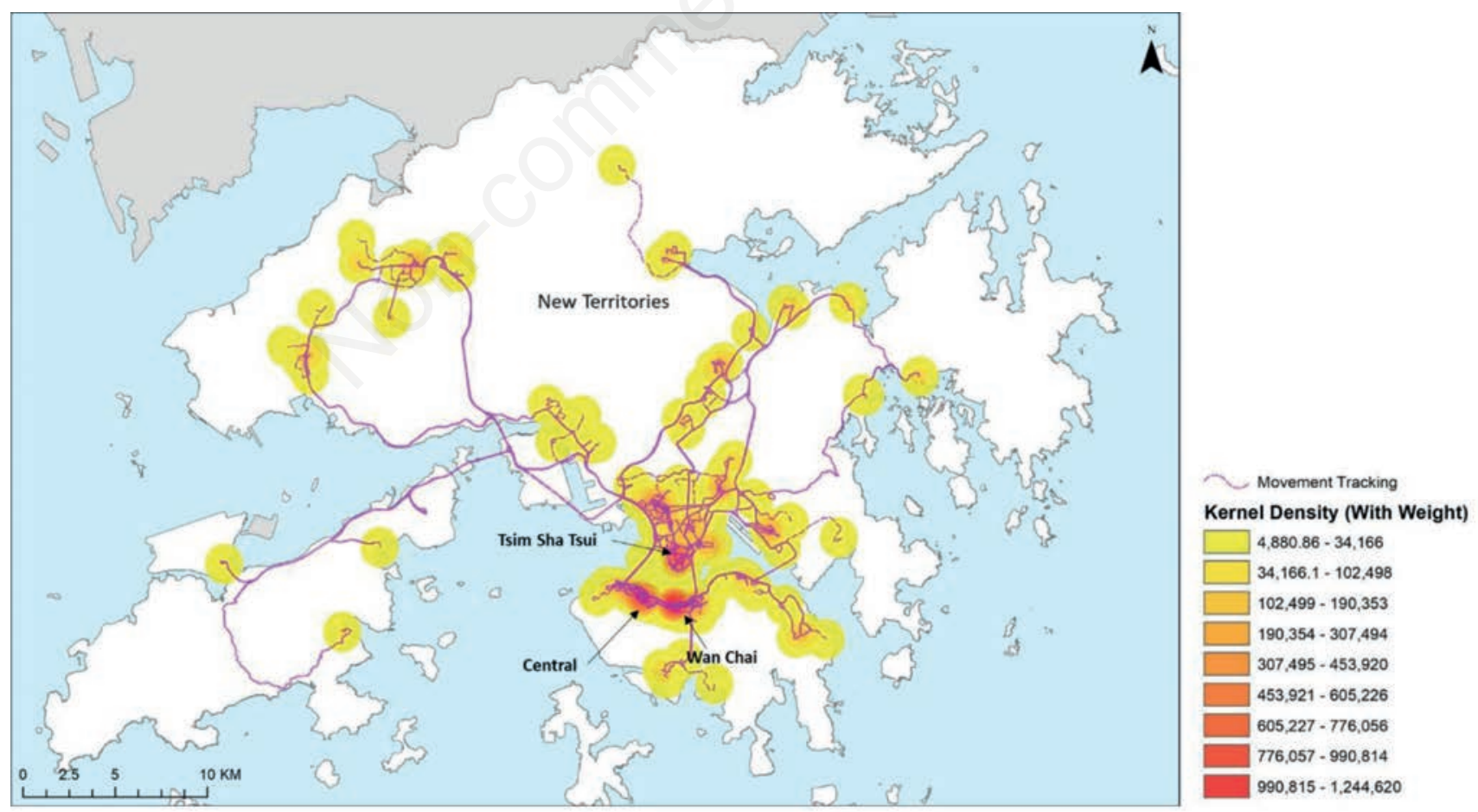

Figure 5. The bar-related COVID-19 infection outbreak in Hong Kong. Movement tracking of infected individuals along major transport routes stratified by three regions: Tsim Sha Tsui, Central and Wan Chai. 


\section{Geographical significance and contact tracing}

The availability of modern mapping software and easy access to frequently updated disease data have made the mapping of major disease outbreaks more feasible. A distribution map of the COVID-19 cases in Hong Kong (Figure 3) provides evidence of the movement effects and behaviours in the local setting. It can be seen that local and imported cases were spatially random, although more imported cases were reported in the business districts along the northern shore of the Hong Kong Island and central part of the Kowloon peninsula. Focusing on period D, the bar-related interactions (Figure 4) analysed here demonstrated that this widespread human-to-human transmission originated from commercial and business districts facilitated by transport networks (Figure 5). Moreover, contract tracing may be replaced with automatic tracking of mobile location data for instant mapping of confirmed infections (Romanillos, et al., 2021; Sui, 2021). The increased possibility of using near real-time data and location intelligence to make informed decisions should thus be an integral part of our epidemic control strategies. This study demonstrates that government actions and interventions can be informed by geographical locations and mobility patterns to avoid total lockdowns which will exert a detrimental impact on the economy.

Visualising spatial variations and movement tracking enables us to consider urban design as a policy instrument to alter social interaction potential. Hong Kong is characterised with mixed-use zoning in which a high-rise residential complex has retail establishments at the lower stories. It can be argued that this kind of mixed-use housing establishment can induce local outbreaks, but the influenced area is limited ( $\mathrm{Li}$ et al., 2020). The option to contain the disease within a limited area without affecting access to daily necessities may reduce the need for travel between regions to reduce the risk of disease spread to other regions.

\section{Conclusions}

Following the first confirmed COVID-19 case that was announced on 23 January 2020, there was much controversy regarding cross-border movement restriction between Hong Kong and China. However, based on the past experiences, especially that gained during the 2003 SARS epidemic, limiting mobility and wearing of surgical facemasks was widely accepted as effective in deterring disease spread. The present study illustrates how different levels of data analysis can be used to visualise disease distribution, as macro-level analysis focuses on the big picture (Figures 2 and 3) and meso-level analysis involves tracking of movements of a specific group (Figures 4 and 5).

As mobile network operators are increasingly offering aggregated big data on population movement (Ienca and Vayena, 2020), and geospatial analysis has undergone rapid technological development in recent years, it is now possible to model disease spread within the community. At the time of completing this article, Hong Kong is considering relaxing some of its social distancing measures and adjusting the limit imposed on group gatherings. It is difficult to predict a suitable time to implement an exit strategy, as relaxing intervention measures too early may result in a new surge of infections. Spatial analysis and visual exploration can enhance the understanding of geographic variation and transmission dynamics at multiple scales, allowing the decision-makers to consider appropriate strategies in a timely manner.

\section{References}

Alvarez FE, Argente D, Lippi F, 2020. A simple planning problem for covid-19 lockdown (No. w26981). National Bureau of Economic Research Working Paper No. 26981. Available from: https://www.nber.org/papers/w26981.pdf Accessed: 10 May 2020.

Carlos HA, Shi X, Sargent J, Tanski S, Berke EM, 2010. Density estimation and adaptive bandwidths: a primer for public health practitioners. Int J Health Geograph 9:39.

Citymapper, 2020. Citymapper Mobility Index, March 2020 September 2021. Available from: https://citymapper.com/cmi/ hongkong Accessed: 10 May 2020.

ESRI, 2020. ESRI China (Hong Kong) homepage. Available from: https://www.esrichina.hk/en-hk/home Accessed: 10 May 2020.

Haklay M, Singleton A, Parker C, 2008. Web mapping 2.0: The neogeography of the GeoWeb. Geography Compass 2:2011-39.

He X, Lau EH, Wu P, Deng X, Wang J, Hao X, Mo X, 2020. Temporal dynamics in viral shedding and transmissibility of COVID-19. Nature Med 1-4.

HKSAR Government, 2020. Latest situation of coronavirus disease (COVID-19) in Hong Kong. Available from: https://chpdashboard.geodata.gov.hk/covid-19/en.html Accessed: 30 April 2020.

Holmes EA, O'Connor RC, Perry VH, Tracey I, Wessely S, Arseneault L, Ford T, 2020. Multidisciplinary research priorities for the COVID-19 pandemic: a call for action for mental health science. Lancet Psychiatr [Epub ahead of print].

Hung LS, 2003. The SARS epidemic in Hong Kong: what lessons have we learned? J R Soc Med 96:374-8.

Ienca M, Vayena E, 2020. On the responsible use of digital data to tackle the COVID-19 pandemic. Nature Med 26:463-4.

Johns Hopkins, 2020. COVID-19 Dashboard. Available from: https://coronavirus.jhu.edu/map.html Accessed: 30 April 2020.

Kraak MJ, 2004. The role of the map in a Web-GIS environment. J Geograph Syst 6:83-93.

Kwan MP, 2000. Interactive geovisualisation of activity-travel patterns using three-dimensional geographical information systems: a methodological exploration with a large data set. Transport Res Part C Emerg Technol 8:185-203.

Lai PC, So FM, Chan KW, 2008. Spatial epidemiological approaches in disease mapping and analysis. CRC Press, Boca Raton, FL, USA.

Lai PC, Wong CM, Hedley AJ, Lo SV, Leung PY, Kong J, Leung GM, 2004. Understanding the spatial clustering of severe acute respiratory syndrome (SARS) in Hong Kong. Environ Health Perspect 112:1550-6.

Lau H, Khosrawipour V, Kocbach P, Mikolajczyk A, Schubert J, Bania J, Khosrawipour T, 2020. The positive impact of lockdown in Wuhan on containing the COVID-19 outbreak in China. J Travel Med 27:taaa037.

Lau JT, Yang X, Leung PC, Chan Lo, Wong E, Fong C, Tsui HY, 2004. SARS in three categories of hospital workers, Hong Kong. Emerg Infect Dis 10:1399.

Li X, Zhou L, Jia T, Peng R, Fu X, Zou Y, 2020. Associating COVID-19 severity with urban factors: a case study of Wuhan. Int J Environ Res Public Health 17:6712.

MacEachren AM, 2004. How maps work: representation, visualization, and design. Guilford Press, New York, NY, USA.

McCloskey B, Heymann DL, 2020. SARS to novel coronavirusold lessons and new lessons. Epidemiol Infection 148. 